\title{
Implizite Berechnung der Grundwasserneubildung (RUBINFLUX) im instationären Grundwasserströmungsmodell SPRING. Eine neue Methodik für regionale, räumlich hochaufgelöste Anwendungen
}

\author{
Harald Zepp $^{1}$ (D) $\cdot$ Christoph König ${ }^{2}$ Julius Kranl ${ }^{1}$ Martin Becker $^{2}$ Barbara Werth B Michael Rathje $^{3}$
}

Eingegangen: 17. Juni 2016 / Überarbeitet: 18. November 2016 / Online publiziert: 12. April 2017

(C) Der/die Autor(en) 2017. Dieser Artikel ist eine Open-Access-Publikation.

Zusammenfassung Das auf Grundlage der Software SPRING erstellte Grundwassermodell für das Stadtgebiet von Düsseldorf $\left(217 \mathrm{~km}^{2}\right)$ gab den Anstoß zur Entwicklung einer neuartigen, robusten Methodik zur Ermittlung der Grundwasserneubildung (RUBINFLUX). Flächendifferenziert und mit täglicher Auflösung waren Neubildungswerte für ein $708 \mathrm{~km}^{2}$ umfassendes Gebiet zu ermitteln. RUBINFLUX ist eine an Lysimeterabflüssen geprüfte Methodik, die bewährte Berechnungsroutinen mit einer e-Funktion für die Sickerwasserabgabe aus dem wasserungesättigten Bodenspeicher kombiniert. Die steuernde Variable ist hierbei die zeitvariante Differenz zwischen der aktuellen und der Bodenwasserspeicherung bei Feldkapazität. Die berechneten Grundwasserneubildungswerte werden in SPRING als Randbedingung 2. Art an der Oberfläche eines jeden Netzelements angesetzt. Die Interaktion der 32 Gewäs-

Harald Zepp

harald.zepp@rub.de

Christoph König

ck@delta-h.de

Julius Kranl

julus.kranl@rub.de

Martin Becker

mb@delta-h.de

Michael Rathje

michael.rathje@duesseldorf.de

1 Geographisches Institut, Ruhr-Universität Bochum, Universitätsstr. 150, 44780 Bochum, Deutschland

2 delta h Ingenieurgesellschaft mbH, Parkweg 67, 58453 Witten, Deutschland

3 Umweltamt, Stadt Düsseldorf, Brinckmannstr. 7, 40225 Düsseldorf, Deutschland sersysteme mit einer Gesamtlänge von $2800 \mathrm{~km}$ wurde instationär mit einer bilanzierenden Randbedingung berücksichtigt. Nach instationärer Kalibrierung anhand von 871 ausgewählten Grundwassermessstellen über 6 Jahre war es möglich, die zeitliche Variation der Grundwasserstände auch an Beobachtungspunkten zufriedenstellend zu modellieren, die von Rhein- und Ruhrwasserständen weitgehend unbeeinflusst sind. In der Implementierung in das Softwarepaket SPRING hat sich RUBINFLUX als geeignet erwiesen, komplexe Hydro-Systeme abzubilden.

\section{High resolution determination of groundwater recharge in transient aquifer modeling}

Abstract The application of the groundwater flow model SPRING to the city of Düsseldorf, Germany $\left(217 \mathrm{~km}^{2}\right)$ as part of a larger hydrological catchment area $\left(708 \mathrm{~km}^{2}\right)$ required developing a new, robust calculation scheme (RUBINFLUX) for groundwater recharge with a high spatial and temporal resolution. RUBINFLUX combines a novel approach for drainage from the unsaturated zone with proven hydrological components. The drainage is calculated as a natural exponential function using the difference between the actual storage and the water storage at field capacity without making use of the Richards equation. The simulated groundwater recharge values at each element of the groundwater mesh were used as the upper boundary condition. After transient calibration of the groundwater flow model against 871 observation wells, the transient variations of the groundwater levels at locations not influenced by river levels were accurately simulated. The integration of RUBINFLUX into SPRING has proved suitable for complex hydrological systems. 
Keywords Groundwater recharge - Unsaturated zone · Water budget - Simulation - Transient - Aquifer modeling · Regional model

\section{Einleitung und Problemstellung}

Die mehrdimensionale instationäre numerische Simulation von Grundwasserströmungen stellt den Stand der Technik bei der Beurteilung von Gefahren und Risiken dar, denen das Grundwasser durch unerwünschte Einträge, Einleitungen oder Entnahmen ausgesetzt ist. In jüngerer Zeit werden derartige Simulationswerkzeuge auch vermehrt genutzt, um Auswirkungen der im Laufe des 21. Jahrhunderts erwarteten Klimaänderungen auf die Aquifere quantitativ abzuschätzen (z. B. Hergesell und Berthold 2008; Lang et al. 2012; Meßer et al. 2013; Regionaler Planungsverband Leipzig-Westsachsen 2013; Ullrich 2013; Gudera und Morhard 2015). Neben Trinkwassergewinnung, Landund Forstwirtschaft werden auch Siedlungsräume von Änderungen der Grundwasserdynamik betroffen sein. Insbesondere durch den möglichen Anstieg des Grundwassers ist gebietsweise mit Schäden an Infrastruktur und Gebäuden sowie Nutzungseinschränkungen zu rechnen. Seit einigen Jahren erarbeitet beispielsweise die Landeshauptstadt Düsseldorf systematisch ein Kataster zu grundwasserbedingten Vernässungsschäden an Gebäuden (Stadt Düsseldorf 2009). Um derartige Gefahren abschätzen und geeignete Schutzmaßnahmen planen zu können, sind flächenhafte, räumlich differenzierte Aussagen unverzichtbar. Zudem wirken sich auch in städtischen Räumen veränderte Grundwasserstände unmittelbar und kurzfristig auf das Hochwassergeschehen infolge von Starkregen und Schneeschmelze aus.

Die Landeshauptstadt Düsseldorf (Nordrhein-Westfalen, Deutschland) erstreckt sich von den Bergischen Randhöhen in Höhen um 130-140 mNN über die Treppe der pleistozänen Rheinterrassen der Niederrheinischen Bucht bis auf Rheinniveau, das im Norden der Stadt bei $30 \mathrm{mNN}$, im Süden bei $35 \mathrm{mNN}$ liegt. Naturräumlich hat die Stadt somit an ihrem östlichen Rand Anteil am Rheinischen Schiefergebirge mit Festgesteinsaquiferen, die in gefalteten devonischen klastischen Gesteinen ausgebildet sind. Der Übergang zu den pleistozänen Terrassen überlagert den bruchtektonischen Rand der Niederrheinischen Bucht; hier liegen bedeutende Porengrundwasserleiter vor, die an ihrer Basis durch tertiäre Tone abgedichtet sind. Aus dem Bergischen Land ziehen einige Gewässer in die Rheinebene herab: Von Norden nach Süden sind dies u. a. die Anger, der Schwarzbach, der Pillebach, die Düssel, der Eselsbach und der Garather Mühlenbach. Meist herrschen influente Zustände, sodass die Gewässer durch leakage Wasser an den Porengrundwasserleiter abgeben. Über den Rand der Niederterrasse fließt instationäres Grund- und infiltrieren- des Oberflächenwasser aus den höher gelegenen Festgesteinsaquiferen dem Porengrundwasserleiter zu. Grundsätzlich sind alle Wasserflüsse auf den Rhein am westlichen Rand und auf die Ruhr am nördlichen Rand ausgerichtet.

Für das Grundwassermodell der Landeshauptstadt Düsseldorf bestand die Herausforderung in der besonderen naturräumlichen und hydrogeologischen Situation im Übergang zwischen Bergland (Festgesteinsaquifer) und Flusslandschaft (Porengrundwasserleiter). Das Stadtgebiet liegt überwiegend im Verbreitungsgebiet des Porengrundwasserleiters (Niederterrasse des Rheins, $313 \mathrm{~km}^{2}$ ). Für dieses waren die Erwartungen an die räumlich differenzierte Modellierung der Grundwasserstände und -ströme besonders hoch. Zeitliche Variationen des Grundwasserspiegels in räumlicher Differenzierung zuverlässig abzubilden gelingt nur unter Berücksichtigung aller quantitativ bedeutsamen instationären Prozesse. Zu ihnen gehört neben Flusswasserständen sowie Wasserentnahmen an Förderbrunnen insbesondere die Grundwasserneubildung. Die Instationarität der Grundwasserneubildung in dem besonders niederschlagsreichen Gebiet des Festgesteinsaquifers $\left(395 \mathrm{~km}^{2}\right)$ gab den Anlass für die Entwicklung von RUBINFLUX, einer Methodik zur flächendifferenzierten Berechnung der instationären Grundwasserneubildung. Sie ist in das Grundwassermodell für den Gesamtraum von $708 \mathrm{~km}^{2}$ so integriert, dass die Neubildung implizit instationär im Modell berechnet und nicht als a priori-Randbedingung bereitgestellt wird.

Erstrebenswert ist eine implizite Berechnung der Grundwasserneubildung, die die Instationarität annähernd so gut abbildet, wie es mit kleinräumig anwendbaren Simulationsmodellen für die ungesättigte Zone möglich ist. Dies schließt eine realitätsnahe Ermittlung der Grundwasserneubildung auch bei Wassergehalten unterhalb der Feldkapazität ein, und es verlangt die Abkehr von zeitlich konstanten Zuflussraten über den Modellrand durch Gebietsvergrößerung. Mit dieser Zielsetzung haben wir die Modellkomponente RUBINFLUX neu entwickelt und in das Grundwasserströmungsmodell SPRING (König et al. 2012) implementiert. Sie ist universell anwendbar und kann in andere Grundwassermodelle integriert werden. Im vorliegenden Beitrag stellen wir die RUBINFLUX-Methodik vor, erläutern Aufbau, Struktur und Kalibrierung des Grundwassermodells für die Landeshauptstadt Düsseldorf und diskutieren abschließend die Bedeutung der Modellkomponente RUBINFLUX für die Lösung der komplexen hydrologischen Aufgabenstellung. 


\section{Berechnung der instationären Grundwasserneubildung}

\section{Verfahrensübersicht}

Die instationäre Grundwasserneubildung aus Klimadaten zu berechnen bedeutet die quantitative Modellierung der Komponenten der Bodenwasserbilanz. Konzeptmodelle stehen hier bodenphysikalischen Modellen gegenüber: Erstere behandeln den Boden als eine Blackbox oder als einen Stapel übereinander geschichteter Speicher, die Wasser nach unten abgeben, sobald sie gefüllt sind. Bodenphysikalische Modelle zerlegen den Boden in eine große Zahl (z. B. 100) übereinanderliegende Kompartimente und rechnen für jedes Kompartiment den Wasserfluss durch den Boden und die Wasserentnahme durch Wurzeln bis nach unten. In der Regel verwenden sie numerische Lösungen für Differenzialgleichungen (z. B. Šimůnek et al. 1998). Nach sorgfältiger Kalibrierung liefern sie sowohl alle gewünschten Wasserhaushaltsgrößen für den gesamten Boden als auch die bodeninternen Flüsse in hoher Auflösung. Wegen des hohen Rechenaufwandes sind sie in der Kombination mit instationären Grundwasserströmungsmodellen für regionale Anwendungen weder in ihren Ausprägungen als 1-dimensionale noch als 3-dimensionale Modelle operationell einsetzbar. In ihren 3D-Versionen können derartige Modelle auf der Hangskala und in Kleineinzugsgebieten eingesetzt werden (z. B. Šimůnek et al. 2006).

Die älteren Konzeptmodelle werden international noch weithin eingesetzt, z. B. Milly (2001), Kim et al. (1996), Laio et al. (2001), Manfreda et al. (2005), Sheffer et al. (2010), Baudena et al. (2012). Häufige Charakteristika dieser Modelle sind, dass sie eine Reduktion der potenziellen (ETo) auf die tatsächliche (ETa) Evapotranspiration beinhalten, sobald der Bodenwasservorrat unter die Feldkapazität $\left(\theta_{\mathrm{FC}}\right)$ absinkt und dass oberhalb von $\theta_{\mathrm{FC}}$ die Wasserabgabe als Grundwasserneubildung zunimmt. In Deutschland werden für die Bestimmung der Bodenwasserbilanz eine ganze Reihe von Methoden angewendet: Glugla et al. (1976), Dörhöfer und Josopait (1980), Renger und Strebel (1980), Schroeder und Wyrwich (1990), Renger und Wessolek (1996), Meßer (1996), Glugla und Fürtig (1997), Bogena et al. (2003), Berthold und Hergesell (2005). In ihren Grundversionen liefern sie Jahreswerte der Grundwasserneubildung und erfüllen somit nicht unsere Anforderungen an Instationarität.

In den letzten Jahren wurden einige Verfahren entwickelt, welche die Grundwasserneubildung innerhalb eines Jahres differenzieren. Dies geschah meist, um die Auswirkungen von Klimaänderungen auf Grundwasserflurabstände abzuschätzen. Meßer et al. (2013) teilen den Jahreswert auf Monate auf und legen dabei einen langjährigen, mittleren Jahresgang zugrunde. Diese Methode ist somit nicht für die Kombination mit instationären Grundwasserströmungsmodellen geeignet, die kurzfristige Schwankungen abbilden sollen. Herrmann et al. (2013) haben mGROWA weiterentwickelt. Die Berechnungen erfolgen tagesweise. Neben der Sickerung oberhalb der Feldkapazität wird präferenzieller Fluss vor Erreichen der Feldkapazität berücksichtigt. Eine Validierung der Methode nehmen die Autoren über den Basisabfluss von Wassereinzugsgebieten vor. An Lysimeterabläufen und grundwasserbürtigem Basisabfluss ist zu beobachten, dass der Abflussrückgang nichtlinear verläuft. Dieser Umstand kann von Modellen, welche die Sickerung erst oberhalb der Feldkapazität zulassen, nicht angemessen beschrieben werden. Gudera und Morhard (2015) begegnen diesem Defizit, indem sie in Zeiten, in denen durch Infiltration dem Boden Wasser zugeführt wird, Sickerung zulassen, noch bevor die Feldkapazität erreicht ist. Die Stärke der Wasserabgabe wird durch das Verhältnis des Bodenwassergehaltes zur nutzbaren Feldkapazität im Wurzelraum gesteuert. Wenn die Feldkapazität als starre Grenze für das Einsetzen der Grundwasserneubildung verwendet wird, können Über- oder Unterschätzungen dieses Parameters zu geringe bzw. zu hohe Grundwasserneubildung zur Folge haben. Im Verdunstungsmodell für die Schweiz TRAIN (Menzel 1999) wird die Wasserleitfähigkeit des Bodens zur Beschreibung der Perkolationsgeschwindigkeit festgesetzt.

Das Grundwassermodell SPRING (König et al. 2012) sollte um einen instationär impliziten Berechnungsansatz für die Grundwasserneubildung erweitert werden, der es erlaubt, im gesamten für die Stadt Düsseldorf hydrologisch relevanten Raum Tageswerte auf der Basis des Witterungsablaufs zu liefern. Hieraus resultierten die Anforderungen an die Methodik: Sie sollte einfach zu parametrisieren sein und wenig Rechnerressourcen beanspruchen. Besonderer Wert wurde darauf gelegt, den in Lysimeterabläufen und bei Trockenwetterabflüssen in Fließgewässern beobachtbaren nichtlinearen Rückgang des Abflusses abzubilden, sobald die Bodenwasserspeicherung auf Werte unterhalb der Feldkapazität sinkt.

\section{Grundwasserneubildung: die RUBINFLUX-Methodik}

RUBINFLUX ist eine Methodik, die bewährte Berechnungsroutinen mit einer neuartigen Formulierung der Sickerwasserabgabe aus dem wasserungesättigten Bodenspeicher kombiniert. Die Bilanzgleichung enthält die in Tab. 1 aufgeführten Komponenten. Sie wird in Tagesschritten für alle Teilflächen des Modells (Netzelemente) bestimmt und ist dort als vertikaler Fluss durch die ungesättigte Zone aufzufassen. 
Tab. 1 Prozesse und Einflussgrößen in RUBINFLUX

\begin{tabular}{|c|c|c|c|}
\hline Prozess & $\begin{array}{l}\text { Berechnete/abgeleitete Ein- } \\
\text { flussgrößen }\end{array}$ & $\begin{array}{l}\text { Einflussgrößen aus } \\
\text { Klimamodell (primäre } \\
\text { Daten) }\end{array}$ & $\begin{array}{l}\text { Gebietseigenschaften } \\
\text { (primäre Daten) }\end{array}$ \\
\hline \multirow[t]{5}{*}{ Direktabfluss (Tageswerte) } & - & $\begin{array}{l}\text { Niederschlagshöhe } \\
\text { (Tagessumme) }\end{array}$ & - \\
\hline & $\begin{array}{l}\text { SCS-Bodenklasse } \\
\text { (Standortparameter) }\end{array}$ & - & Bodenartenklasse (BK50) \\
\hline & $\begin{array}{l}\text { SCS-Landnutzungsklasse } \\
\text { (Standortparameter) }\end{array}$ & - & ATKIS/Realnutzungskartierung \\
\hline & $\begin{array}{l}\text { Hangneigung } \\
\text { (Standortparameter) }\end{array}$ & - & $\begin{array}{l}\text { Relief (DGM10; Geländehöhe- und } \\
\text {-neigung) }\end{array}$ \\
\hline & $\begin{array}{l}\text { Bodenfeuchteklasse zu Ta- } \\
\text { gesbeginn (täglich) }\end{array}$ & $\begin{array}{l}\text { Niederschlagshöhe der } \\
5 \text { Vortage (Tagessum- } \\
\text { men) }\end{array}$ & $\begin{array}{l}\text { Beginn und Ende der Vegetations- } \\
\text { phase }\end{array}$ \\
\hline \multirow[t]{3}{*}{$\begin{array}{l}\text { Interzeption/Interzeptionsverdunstung } \\
\text { (Tageswerte) }\end{array}$} & $\begin{array}{l}\text { Grasreferenzverdunstung } \\
\text { (ETo) } \\
\text { (Tageswerte) }\end{array}$ & $\begin{array}{l}\text { Windgeschwindigkeit } \\
\text { ( } 2 \mathrm{~m} \text {, Tagesdurch- } \\
\text { schnitt), Lufttempe- } \\
\text { ratur ( } 2 \mathrm{~m} \text {, Tagesdurch- } \\
\text { schnitt), Sonnenschein- } \\
\text { dauer (Tagessumme), } \\
\text { Dampfdruck (Tages- } \\
\text { durchschnitt) }\end{array}$ & - \\
\hline & $\begin{array}{l}\text { Verhältnis ETmax/ETo } \\
\text { (Standortparameter) }\end{array}$ & - & $\begin{array}{l}\text { Bodenartenklasse aus BK50, } \\
\text { ATKIS/Realnutzungskartierung }\end{array}$ \\
\hline & $\begin{array}{l}\text { Interzeptionshöhe } \\
\text { (Tagessumme) }\end{array}$ & $\begin{array}{l}\text { Niederschlagshöhe } \\
\text { (Tagessumme) }\end{array}$ & Blattflächenindex (LAI) \\
\hline \multirow[t]{4}{*}{ Evapotranspiration (Tageswerte) } & $\begin{array}{l}\text { Grasreferenzverdunstung } \\
\text { (ETo) } \\
\text { (Tageswerte) }\end{array}$ & $\begin{array}{l}\text { Windgeschwindigkeit } \\
\text { (10 m, Tagesdurch- } \\
\text { schnitt), Lufttempera- } \\
\text { tur ( } 2 \mathrm{~m} \text {, Tagesdurch- } \\
\text { schnitt), Sonnenschein- } \\
\text { dauer (Tagessumme), } \\
\text { Dampfdruck (Tages- } \\
\text { durchschnitt) }\end{array}$ & Geogr. Breite \\
\hline & $\begin{array}{l}\text { Verhältnis } \mathrm{ET}_{\max } / \mathrm{ETo} \\
\text { (Standortparameter) }\end{array}$ & - & $\begin{array}{l}\text { Bodenartenklasse aus BK50, } \\
\text { ATKIS/Realnutzungskartierung }\end{array}$ \\
\hline & $\begin{array}{l}\text { Interzeptionshöhe } \\
\text { (Tageswerte) }\end{array}$ & $\begin{array}{l}\text { Niederschlagshöhe } \\
\text { (Tagessumme) }\end{array}$ & $\begin{array}{l}\text { Blattflächenindex } \\
\text { (LAI) }\end{array}$ \\
\hline & 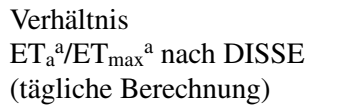 & - & $\begin{array}{l}\text { Wassergehalte bei Feldkapazität } \\
\text { und PWP aus BK50 }\end{array}$ \\
\hline $\begin{array}{l}\text { Sickerwasserabgabe unterhalb } 13 \mathrm{dm} \\
\text { (Tageswerte) }\end{array}$ & $\begin{array}{l}\text { Verhältnis Sickerwasser } \\
\text { (Tagessumme)/täglichem } \\
\text { Bodenwassergehalt nach } \\
\text { RUBINFLUX-Funktion }\end{array}$ & - & $\begin{array}{l}\text { Wassergehalt bei Feldkapazität aus } \\
\text { BK50 }\end{array}$ \\
\hline
\end{tabular}

${ }^{\mathrm{a}}$ vermindert um Interzeptionsverdunstung

Der Bodenwassergehalt zu Tagesbeginn $\mathrm{W}_{\mathrm{i}}$ wird berechnet nach

$W_{i}=W_{i-1}+P_{i-1}-D R_{i-1}-I_{i-1}-E T_{i-1}-S W_{i-1}$
$E T_{i-1} \quad$ Evapotranspiration,

$S W_{i-1} \quad$ Sickerwasserabgabe an den tieferen Untergrund am Ende des vorausgegangenen Tages; alle Angaben in $\mathrm{mm}$.

Das USDA-SCS-Verfahren nach DVWK (1991) und Williams (1995) in der Modifikation für Mitteleuropa nach Becker und Rosemann (1995) diente zur Ermittlung des Direktabflusses, des Niederschlagsanteils, der nach einem Niederschlagsereignis (hier angenähert durch Tagesnieder- 
schläge) nicht im Gebiet zurückgehalten, um zu verdunsten oder an den Grundwasserkörper abgegeben zu werden, sondern den Vorflutern direkt zugeführt wird. Mit dem SCSVerfahren wird rechnerisch der Direktabfluss ohne Differenzierung des Fließwegs auf der Bodenoberfläche oder als hypodermischer Abfluss und ohne Berücksichtigung der Weglängen abgeschätzt. In der Realität überwiegt beim Direktabfluss die laterale Richtungskomponente, doch werden diese im Grundwassermodell ausschließlich bei den Interaktionen zwischen Vorfluter und Grundwasser berücksichtigt. Aus der Niederschlagssumme der vorangegangenen Tage, Bodeneigenschaften und Landnutzung wird das Speichervermögen ermittelt und dann dem aktuellen Tagesniederschlag gegenübergestellt. Oberflächenabfluss infolge Versiegelung (Befestigung) wurde berücksichtigt.

Die Interzeptionsspeicherung folgt Hoyningen-Huene in DVWK (1983). Die potenzielle Verdunstung wird nach Monteith-Rijtema in der Version der Gras-ReferenzVerdunstung nach FAO (2012) berechnet und kulturartenspezifisch durch Bestandskoeffizienten angepasst. Das zu Tagesbeginn im Interzeptionsspeicher verfügbare Wasser wird zu Lasten der potenziellen Verdunstung des Tages vermindert. Die Disse-Funktion (Disse 1995) dient der Reduktion der potenziellen Verdunstung auf die tatsächliche Transpiration (Gl. 2). Sinkt der Wassergehalt im Wurzelraum des Bodens, so ist die Transpiration reduziert.

$$
\frac{E T_{a}{ }^{*}}{E T_{\max }{ }^{*}}=\frac{1-e^{-r x}}{1+e^{-r x}-2 e^{-r}}
$$

$$
\begin{array}{ll}
E T_{a}{ }^{*} & \begin{array}{l}
\text { tatsächliche Evapotranspiration, vermindert } \\
\text { um die Interzeptionsverdunstung, }
\end{array} \\
E T_{\max i}{ }^{*} & \begin{array}{l}
\text { maximale Evapotranspiration, vermindert um } \\
\text { die Interzeptionsverdunstung, }
\end{array} \\
\mathrm{r} & =5, \\
x & \text { relativer Bodenwassergehalt. }
\end{array}
$$$$
x==\frac{\theta_{i}-\theta_{P W P}}{\theta_{F C}-\theta_{P W P}}
$$

$$
\begin{array}{ll}
\theta_{i} & \text { Bodenwassergehalt am Tag } i[\%], \\
\theta_{P W P} & \begin{array}{l}
\text { Bodenwassergehalt am Permanenten Welke- } \\
\text { punkt [\%], }
\end{array} \\
\theta_{F C} & \text { Bodenwassergehalt bei Feldkapazität [\%]. }
\end{array}
$$

Ebenso wie sich die Transpiration mit abnehmender Bodenfeuchte nichtlinear verringert, vermindert sich die Durchsickerung des Bodens. Wir berücksichtigen, dass nicht erst ab der Überschreitung der Feldkapazitätsgrenze Wasserabgabe aus dem Boden stattfindet. Diese Zusammenhänge drücken Bodenhydrologie und Bodenphysik mit der Richards-Gleichung aus. Numerische Lösungen dieser nichtlinearen Differenzialgleichung bilden Kernelemente bodenphysikalisch fundierter Simulationsmodelle für den Bodenwasserhaushalt (z. B. Šimůnek et al. 1998, 2006). Wegen des hohen Rechenaufwandes kann diese Methode nicht für sämtliche Teilflächen implizit im Grundwassermodell angewendet werden, sodass wir eine vereinfachte Formulierung gewählt haben. Die tägliche Sickerwasserabgabe $\left(S W_{\mathrm{i}}\right)$ wird nach Gleichungen $4-6$ berechnet (Abb. 1).

$$
\begin{aligned}
& S W_{i}=\left\{\begin{array}{l}
s_{i} \cdot x \cdot d, x>1 \\
s_{i} \cdot d, x \leq 1
\end{array}\right. \\
& s_{i}=1 /\left(1+e^{-x}\right) \\
& x=W C S_{i} \cdot d^{-1}-\theta_{F C}=\theta_{i}-\theta_{F C}
\end{aligned}
$$

$\begin{array}{ll}S W_{i} & \begin{array}{l}\text { Sickerwasserabgabe an den tieferen Unter- } \\ \text { grund [mm] }\end{array} \\ W C S_{i} & \text { Bodenwassergehalt zu Tagesbeginn [mm] } \\ \theta_{F C} & \text { Feldkapazität [\%] } \\ s_{i} & \text { Sickerungsfaktor [-] } \\ d & \text { Simulationstiefe [dm] }\end{array}$

Standardmäßig ist die Simulationstiefe auf $13 \mathrm{dm}$ eingestellt. Bei Bodenfeuchtewerten nahe der Feldkapazität tritt eine kräftige Sickerwasserabgabe auf (Abb. 1a), oberhalb von $\theta_{F C}+1$ Vol.- $\%$ ist sie sogar sehr stark, aber niemals so stark, dass - wie in vereinfachten Ansätzen, welche die Feldkapazität als absolute Grenze ansetzen - alles überschüssige Wasser sofort nach unten abgeschlagen wird. Mit abnehmender Feuchte lässt unsere Formulierung auch unterhalb der FK noch Wasserabgabe zu. Die Wasserabgabe aus dem $13 \mathrm{dm}$ mächtigen Boden definieren wir als Grundwasserneubildung.

Beispielhaft zeigt sich die Entleerungsfunktion für einen Bodenspeicher mit einer Feldkapazität von 26 Vol.-\% (Abb. 1b). Ausgehend von einem Wassergehalt von 28 Vol.-\% sinkt - ohne Wasserverlust durch Evapotranspiration - der Bodenwassergehalt, nach einem Monat sind 22,6 Vol.-\% erreicht. Das Ausmaß der Wasserabgabe nimmt also nichtlinear $a b$.

\section{Prüfung der RUBINFLUX-Methodik}

Die neu entwickelte Entleerungsfunktion sowie die Gesamtmethodik von RUBINFLUX wurden vor ihrer Integration in SPRING geprüft, um sicherzustellen, dass sie Grundwasserneubildungsberechnungen zuverlässig und mit der geforderten zeitlichen Auflösung erlauben. Die Prüfung erfolgte an mehrjährigen Messreihen der GrünlandLysimeter in Rheindahlen (Löss; NEW - NiederrheinWasser GmbH, Mönchengladbach) und St. Arnold (Sand; Landesamt für Natur, Umwelt und Verbraucherschutz NRW 
Abb. 1 a RUBINFLUX-Entleerungsfunktion für den Bodenwasserspeicher und $\mathbf{b}$ modellhafte Entleerung eines Bodenspeichers von 1,3 m Mächtigkeit, ohne Verdunstungseinfluss

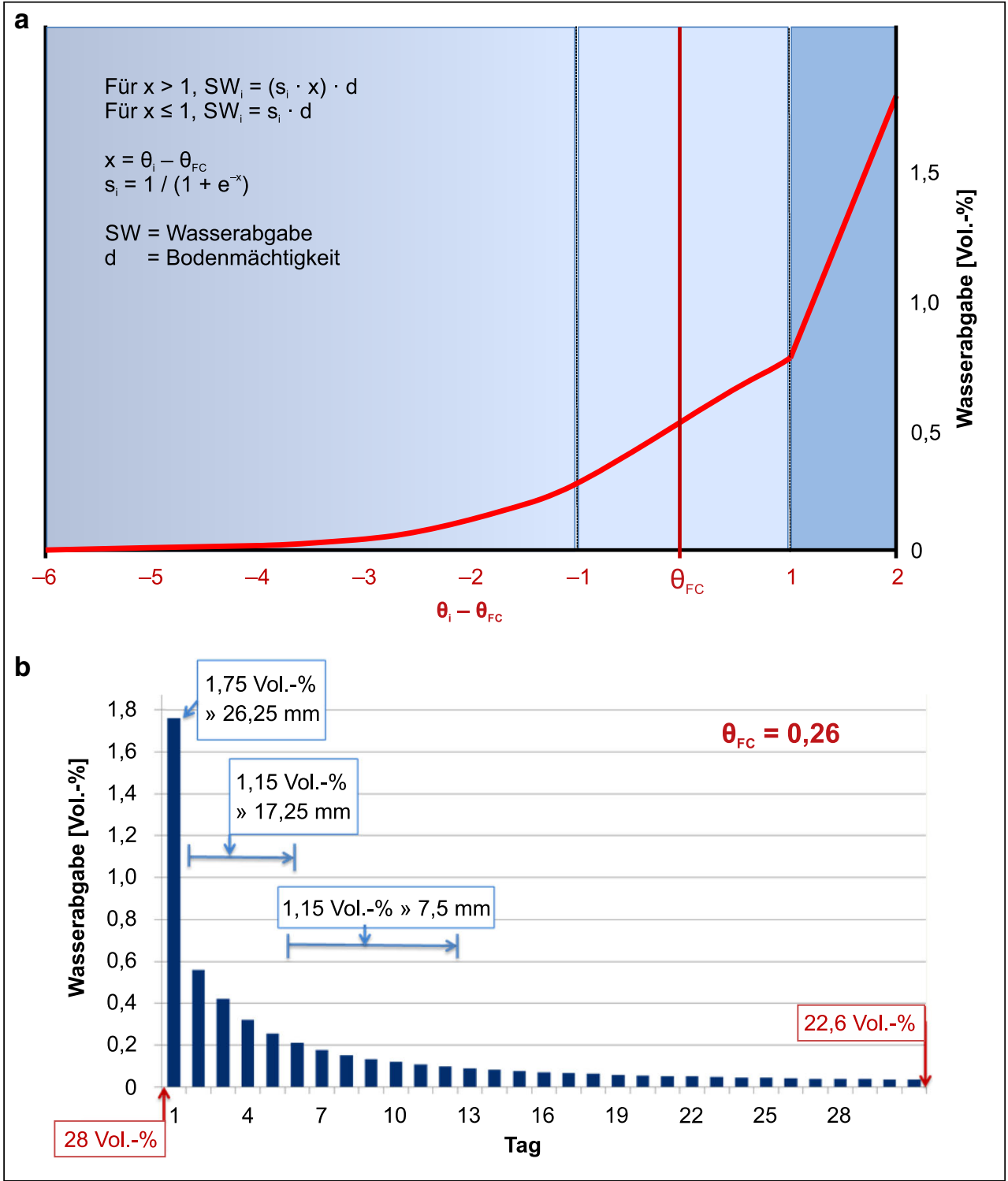

(LANUV), Recklinghausen; Harsch et al. 2009). Angaben für die Feldkapazität wurden den Lysimeterbeschreibungen entnommen (Schumacher und Wellens 1993; Klein 2000). Die Kalibrierung beschränkte sich auf die Anpassung des Bestandskoeffizienten der Verdunstungsgleichung, um Unterschiede im Transpirationsverhalten der beiden grasbewachsenen Lysimeter auszugleichen. Im Lysimeter St. Arnold wird das Sickerwasser in 3,5 m Tiefe registriert; da RUBINFLUX auf eine Simulationstiefe von 1,3 m eingestellt ist, wurden hier die simulierten Tageswerte um 6 Tage gegenüber den gemessenen verschoben, um der wegen der längeren Sickerstrecke verzögerten Wasserabgabe des Lysimeters Rechnung zu tragen.

Die nichtlineare Abnahme der gemessenen Durchsickerungshöhen (Abb. 2) ist an beiden Standorten durch die Simulation gut wiedergegeben. Der Korrelationskoeffizient beträgt am Standort Rheindahlen 0,86, am Standort St. Arnold 0,72. Insgesamt werden Zeiträume mit Wasserabgabe aus dem Lysimeter zufriedenstellend abgebildet und auch absolute Höhen gut getroffen. Das Ergebnis der Prüfung der RUBINFLUX-Methodik ermutigte - insbesondere angesichts des minimalen Aufwandes bei der Anpassung der Berechnungsmethode an die standörtlichen Verhältnisse -, es in die Software SPRING zu integrieren. 


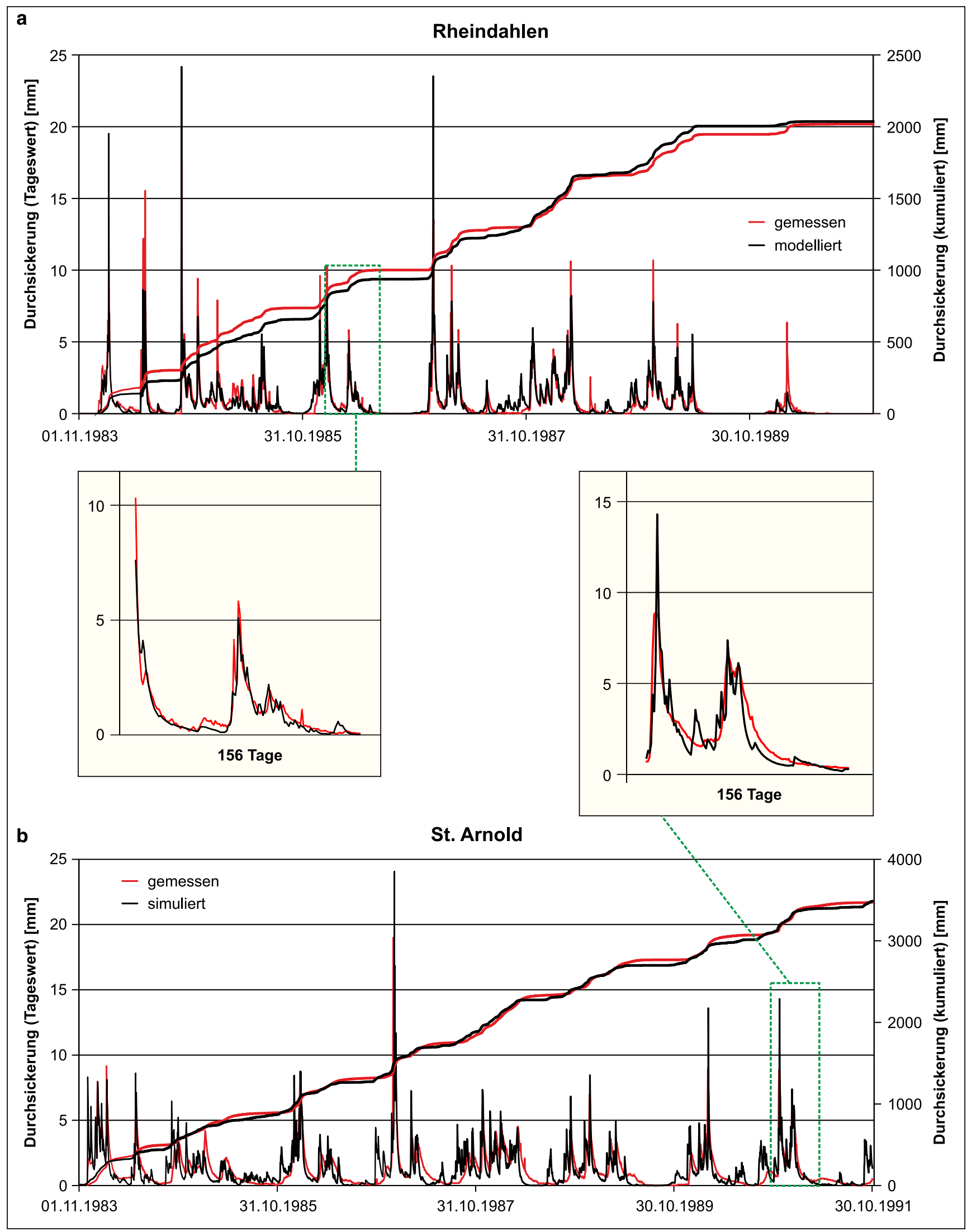

Abb. 2 Gemessene und simulierte Durchsickerung (jeweils Tageswerte und kumuliert); a Lysimeter Rheindahlen (Löss; $\theta_{F C}: 38$ Vol.-\%; Bestandskoeffizient von ETo: 0,83); b Lysimeter St. Arnold (Sand; $\theta_{F C}: 15,7$ Vol.-\%; Bestandskoeffizient von ETo: 0,7) 
Abb. 3 a Flächenhafte Ausdehnung des GW-Modells und b schematischer Vertikalschnitt des GW-Modells

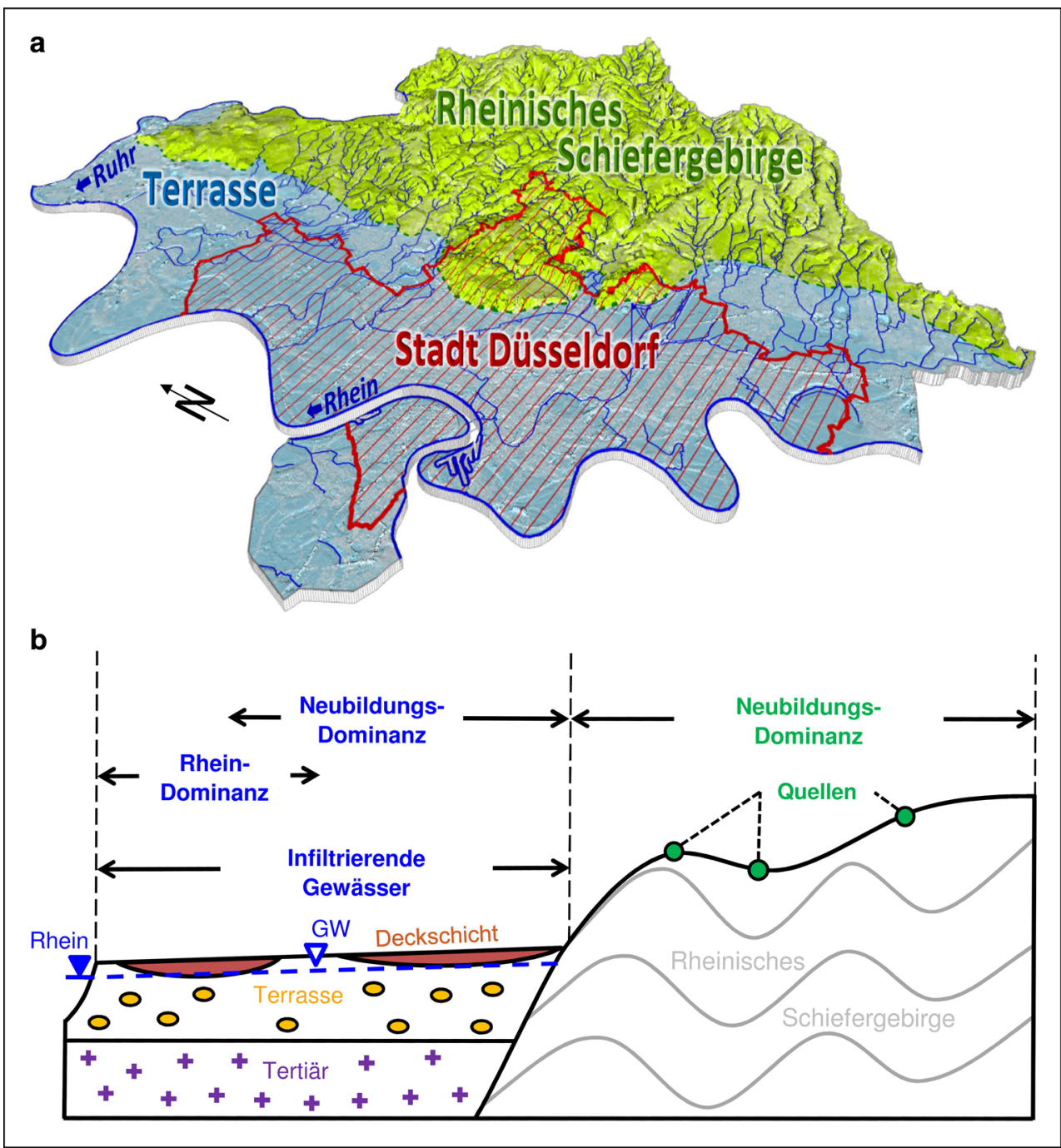

\section{Aufbau, Struktur und Kalibrierung des Grundwassermodells}

\section{Modellaufbau}

Das Grundwassermodell wird im Westen durch den Rhein, im Osten durch die Wasserscheide im Bergischen Land in Höhen von bis zu $300 \mathrm{~m}$ sowie im Norden durch die Ruhr begrenzt. Ihre Wasserspiegelschwankungen wirken sich untergeordnet zu den dominanten Einflüssen des Rheinwasserspiegels auf das Grundwasser der nördlichen Stadtteile aus. Der Grundwasserzufluss über den östlichen Terrassenrand und durch „leakage“ aus den Bächen aus dem Bergischen Land beeinflusst die Grundwasserstände des Lockergesteinsaquifers mit zunehmender Entfernung von Rhein und Ruhr immer stärker. In dem $708 \mathrm{~km}^{2}$ großen Gebiet variiert der Jahresniederschlag zwischen $800 \mathrm{~mm}$ in der Rheinebene und mehr als $1200 \mathrm{~mm}$ im Bergischen Land.
Wasserstauende tertiäre Tone begrenzen den Porengrundwasserleiter an seiner Basis. Im Bergland östlich der Verbreitungsgrenze der Tertiär-Tone wurde die Modellunterfläche aus der Geländemorphologie abgeleitet. Der Modellrand verläuft im Westen entlang des Rheins und im Norden entlang der Ruhr mit der Randbedingung 1. Art (Festpotenzial; Dirichlet). Der östliche und südliche Modellrand wurde anhand von (oberirdischen) Einzugsgebieten von Oberflächengewässern (vgl. Abb. 3) als Wasserscheide (Randbedingung 2. Art mit $\mathrm{q}=0$ ) festgelegt. Die Grundwasserströmung vom Kluft- in den Porengrundwasserleiter erfolgt als Funktion des Potenzials und der k-Werte. Bei der Generierung des Elementnetzes waren diverse Zwangsgeometrien zu berücksichtigen. Neben den Brunnenstandorten, den Grundwassermessstellen, den voll- und teilsperrenden Bauwerken und den Stillgewässerflächen wurde auch das Oberflächengewässersystem als Zwangsgeometrie verwendet. Verteilt auf ca. $32 \mathrm{maß-}$ gebende Teilsysteme ergibt sich im Modell eine Länge 
von ca. $2800 \mathrm{~km}$. Zur Abbildung der Interaktion zwischen den Fließgewässern und dem Grundwasser sind an den die Gewässer repräsentierenden Modellknoten jeweils der Wasserstand im Gewässer und ein Leakage-Koeffizient zur Beschreibung des Sohlwiderstands des Gewässerabschnitts anzugeben (Randbedingung 3. Art; Cauchy). Die Wasserstände wurden aus dem Geländemodell abgeleitet, die Leakage-Koeffizienten anhand von Grundwassermessstellen unter Einbeziehung der berechneten Flurabstände kalibriert. Die eingesetzte Modellierungssoftware SPRING ermöglicht eine fließrichtungsbezogene Vernetzung der das Gewässersystem beschreibenden Modellknoten. Auf diese Weise wird die maximal mögliche Menge, die über einen Gewässerabschnitt in das Grundwasser versickern kann, auf die oberstromig zugeflossene Menge limitiert. Da im Düsseldorfer Stadtgebiet eine Vielzahl von Gewässerabschnitten ganzjährig oder saisonal über dem Grundwasser liegen, kann dort mit dieser Modellierungstechnik die Zusickerung ins Grundwasser in Abhängigkeit des Dargebots instationär quantifiziert und somit limitiert werden. In länger andauernden Trockenperioden kann hierdurch auch ein temporäres Trockenfallen einzelner Gewässerabschnitte abgebildet werden. Über eine Parametrisierung der vernetzten Gewässerabschnitte kann zudem der Wasserstand aus der aktuellen Abflussmenge automatisch abgeschätzt werden. Hierzu wird für jeden Abschnitt das Sohlgefälle aus dem Wasserstand abgeleitet. Die erforderlichen Angaben zum benetzten Umfang und zum Manning-Strickler-Beiwert wurden für alle Gewässerabschnitte stückweise konstant angenommen. Das 2D-Modell besteht aus rund 310.000 Knoten und 382.000 Elementen. Im Bergland besitzen die Elemente eine Kantenlänge von bis zu 150 m, im Gebiet des Porenrundwasserleiters wurden die Elemente mit Kantenlängen von maximal $40 \mathrm{~m}$ erzeugt. Im Übergangsbereich zwischen beiden Teilräumen betragen die Kantenlängen rund $80 \mathrm{~m}$, um einen harmonischen Übergang sicherzustellen.

Die nach der RUBINFLUX-Methodik implizit errechnete Wasserabgabe aus den ungesättigten oberen $13 \mathrm{dm}$ wird der Grundwasserneubildung gleichgesetzt und tritt in jedem Element des Modells in den Grundwasserkörper ein, sowohl im Festgesteins- als auch im Lockergesteinsgebiet. Die den Wurzelraum durchsickernden Wassermengen werden in der Regel in Abhängigkeit von der Durchlässigkeit des Untergrunds und der Flurabstände während der Passage durch die ungesättigte Zone in ihrer Amplitude gedämpft. Dieser Effekt kann in der Grundwassermodellierung nur bei 3D-Modellen und dann nur bei sehr hoch aufgelöster vertikaler Diskretisierung nachgebildet werden. Da die erforderliche vertikale Diskretisierung im regionalen Maßstab nicht praxistauglich umgesetzt werden kann und um solche Effekte auch bei 2D-Modellen zu berücksichtigen, wurde bei der Programmierung die Möglichkeit eingeräumt, die
Ausgabe der berechneten Tagessummen der Grundwasserneubildung als Mittelwert über eine frei wählbare Anzahl von Tagen zu exportieren. Hierdurch können starke, kurzzeitig auftretende Schwankungen in der Sickerwasserrate gedämpft werden, ohne die Wasserbilanz über den zu mittelnden Zeitraum zu verfälschen. Für das vorliegende Modell ergab eine Mittelung der Grundwasserneubildungsraten auf 10-Tageswerte eine für die Modellrechnungen gut geeignete Dämpfung. Die Oberflächengewässer nehmen in Abhängigkeit vom Gradienten des hydraulischen Potenzials zwischen dem Aquifer und dem Wasserstand im Gewässer zufließendes Wasser auf, führen es ab oder füllen den Grundwasserkörper auf. Zusätzlich erhalten die Gewässer den zuvor für jedes Element des Modells nach dem SCSVerfahren berechneten schnellen Abfluss. Dieser schnelle Abfluss fließt aus dem, jedem Gewässerknoten zugeordneten lokalen Einzugsgebiet ohne Verzögerung zu.

Für die durch Gewässer definierten Modellränder wurden die Wasserstände auf Basis der Tageswerte am Pegel Düsseldorf (Rhein) sowie Werden und Mülheim (Ruhr) definiert bzw. interpoliert.

Die Modellgenerierung berücksichtigt das Oberflächengewässersystem als Zwangsgeometrie.

\section{Modelleingangsdaten und -kalibrierung}

Das Untersuchungsgebiet wurde auf der Basis von 28 Niederschlagsstationen in ebenso viele Niederschlagszonen eingeteilt, die somit im Mittel Flächen von jeweils $25 \mathrm{~km}^{2}$ repräsentieren. Aus ihnen ergab sich die Zuordnung der täglichen Niederschlagshöhen zu den Netzelementen ohne räumliche Interpolation. Für die Netzelemente waren ferner konstante Kennwerte wie Geländerelief, Feldkapazität und instationäre Parameter für die Berechnung der Verdunstung (Sonnenscheindauer, Lufttemperatur, Luftfeuchtigkeit, Windgeschwindigkeit) anzugeben. Für alle Flächennutzungen außer Acker wurden aus den im Verfahren BAGLUVA (Bundesanstalt für Gewässerkunde 2003) angegebenen Rechenvorschriften zunächst mittlere jährliche Bestandskoeffizienten für die zuvor definierten Landnutzungs- und Bodenklassen des Untersuchungsgebiets abgeleitet (zur Herkunft der umfangreichen Datenbestände siehe Danksagung). Die jeweiligen Jahreswerte waren Grundlage für die Ermittlung von Bestandskoeffizienten für die Vegetationsphase und für die Vegetationsruhe. Im Modell wurden für Ackerflächen monatliche Bestandskoeffizienten aus empirischen Untersuchungen an der Lysimeteranlage Brandis ohne die dort angegebene Differenzierung in Halmund Hackfrüchte übernommen (ATV-DVWK 2002). Die vorgenannten Daten und ihre Aufbereitung waren für die RUBINFLUX-Methodik erforderlich (vgl. Tab. 1). Der Befestigungsgrad lag für weite Teile des Stadtgebiets in $20 \%$-Intervallen vor; im übrigen Gebiet wurde er aus 
der Flächennutzung geschätzt. Entsprechend wurde die Evapotranspiration vermindert und der Oberflächenabfluss erhöht.

Das Modell wurde durch die Anpassung der Parameter für die Grundwasserkörper zunächst auf eine turnusmäßige Stichtagsmessung vom Oktober 2006 stationär kalibriert. Dieser Zeitpunkt kam einem Zustand mit quasi-mittleren Grundwasser- und Rheinwasserständen am nächsten. Dazu mussten Jahresmittelwerte der Grundwasserneubildung als Modellrandbedingung vorgegeben werden. Die stationäre Kalibrierung lieferte den Ausgangszustand für die instationäre Kalibrierung. Aufgrund der Tatsache, dass das Grundwasserregime im westlichen Stadtgebiet maßgeblich von der Dynamik des Rheinwasserstands und nicht vom
Niederschlag im Einzugsgebiet geprägt ist, während sich die Einflüsse im östlichen Stadtgebiet genau entgegengesetzt verhalten, treten mittlere Grundwasserstände für das gesamte Stadtgebiet nur sehr selten und somit auch nur zufällig an Tagen mit Stichtagsmessungen auf. Daher sind Stichtagsmessungen in Gebieten wie dem vorliegenden erst im Kontext einer instationären Kalibrierung nachhaltig bewertbar. Die instationäre Kalibrierung des Modells erfolgte für die Wasserwirtschaftsjahre 2005-2010 in Tagesschritten. Dabei wurden als zeitlich variierende Randbedingungen die Wasserstände von Rhein und Ruhr (Tageswerte), die Grundwasserentnahmen (Sanierungsbrunnen, Bauwasserhaltungen und Trinkwasserentnahmen), voll- und teilsperrende Bauwerke jeweils zum Fertigstellungsdatum und
Abb. 4 Mittlere jährliche Grundwasserneubildungsraten (WWHJ 2005-2010)

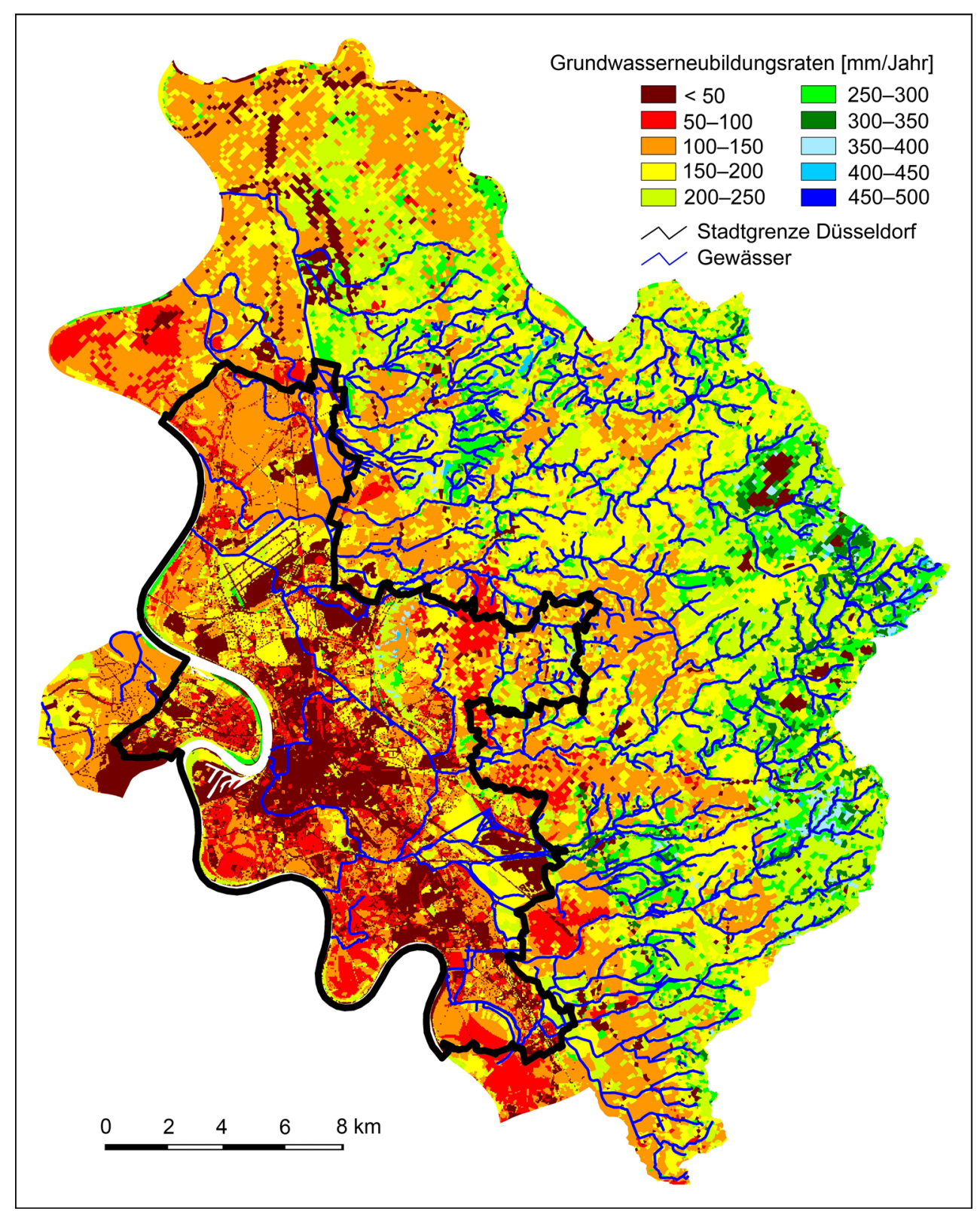


die Grundwasserneubildung (10-Tagesmittel) angesetzt. Weder bei der stationären noch bei der instationären Kalibrierung wurden die aus der RUBINFLUX-Methodik im Grundwassermodell berechneten Wasserbilanzen adjustiert. Die instationäre Kalibrierung erfolgte anhand von 370 Grundwassermessstellen mit täglichen und an $401 \mathrm{mit}$ monatlichen Wasserstandsaufzeichnungen.

\section{Modellergebnisse und -validierung}

Für die stationäre Kalibrierung des Grundwassermodells auf eine Stichtagsmessung von Oktober 2006 wurde die nach der Methode RUBINFLUX auf der Grundlage der flächendifferenzierten Standortparameter und Klimaparameter ermittelte, durchschnittliche Grundwasserneubildung von $157 \mathrm{~mm} / \mathrm{Jahr}$ (98 mm/Jahr im Stadtgebiet) herangezogen. Sie ist zunächst Ergebnis der modellinternen Wasserbilanzrechnungen nach der RUBINFLUX-Methodik und andererseits Ausgangspunkt für die Kalibrierung der aquiferbezogenen Modellparameter. Die räumliche Verteilung (Abb. 4) zeigt, dass die Methode realistische Werte liefert. Bei jährlichen Niederschlagshöhen bis $1200 \mathrm{~mm}$ werden über $400 \mathrm{~mm}$ erwartet, im teilversiegelten Innenstadtgebiet mit Niederschlägen um $800 \mathrm{~mm}$ sind es dagegen unter $50 \mathrm{~mm}$. Eine Gegenüberstellung der berechneten und gemessenen Grundwasserstände an 871 Grundwassermess- stellen hat eine mittlere absolute Abweichung von 0,28 m ergeben.

Allerdings erweist sich der Vorteil der auf instationäre Prozesse zielenden Methodik erst in der Kombination mit dem Grundwassermodell. Die für jedes Netzelement berechnete Grundwasserneubildung tritt im Modell als Grundwasser je nach Stärke und Richtung des hydraulischen Potenzialgefälles entweder direkt in den Porengrundwasserleiter der Niederterrasse ein oder zusammen mit dem Direktabfluss in die Vorfluter über. Ein Kriterium für die Modellgüte ist also, ob der mittlere Abfluss der Vorfluter aus dem niederschlagsreichen Bergland zufriedenstellend bilanziert werden kann. Dies wurde an Pegeleinzugsgebieten geprüft: Das hier exemplarisch vorgestellte $9 \mathrm{~km}^{2}$ große Einzugsgebiet des Pegels Lochbachmündung beinhaltet im Wesentlichen den nördlichen Teil der Siedlungsgebiete der Stadt Solingen mit einem geschätzten Befestigungsgrad von $50 \%$. Am Pegel beträgt der Mittelwert der gemessenen Tagesminima 50 1/s und das Tagesmittel 150 1/s. Unsere Rechnungen im Grundwassermodell ergaben für das Einzugsgebiet dieses Pegels einen mittleren grundwasserbürtigen Abfluss von 60 1/s und einen mittleren Tagesabfluss (Summe aus Grundwasserneubildung (RUBINFLUX) und schnellen Abflusskomponenten (SCS)) von 1201/s. Die zeitlichen Verläufe der berechneten und gemessenen (Abb. 5a) Tagesabflüsse zeigen sehr gute Übereinstimmung in der zeitlichen Dynamik, obschon im Modell Verzögerungseffekte bei den

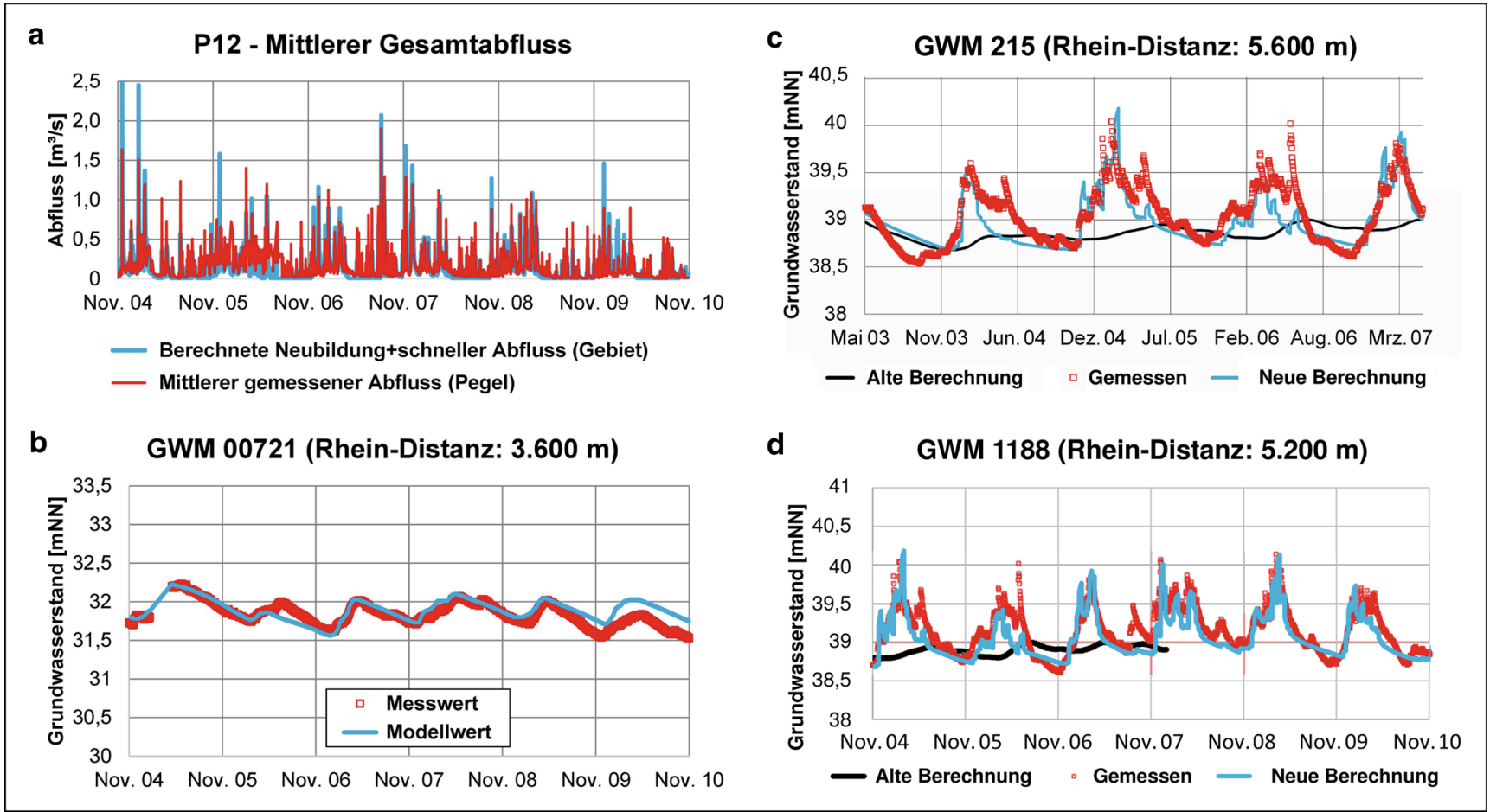

Abb. 5 a Gemessene und berechnete mittlere Tagesabflüsse am Pegel P12/Lochbachmündung (6 Jahre); b gemessene und berechnete Grundwasserstände in größerer Entfernung zum Rhein; c und d gemessene und berechnete Grundwasserstände mit (neu) und ohne (alt) Berücksichtigung instationärer Grundwasserneubildung (4 Jahre) 
schnellen Abflusskomponenten nicht berücksichtigt wurden.

Die Kalibrierung des Modells an Grundwasserganglinien von 370 Loggern in den Wasserwirtschaftsjahren 2005-2010 ergab eine mittlere Abweichung von nur $3 \mathrm{~cm}$. Mit zunehmender Entfernung vom Rhein ist nun der niederschlagsbedingte beobachtete Grundwassergang gut vom Grundwassermodell nachgebildet (Abb. 5b-d).

Für einen kürzeren Zeitraum sind in Abb. $5 c$ und d die gemessenen den berechneten Grundwasserständen mit und ohne Berücksichtigung von instationärer Neubildung gegenübergestellt. Während die Berechnung mit Berücksichtigung der instationären Neubildung die Dynamik der gemessenen Ganglinie nachbilden kann, ist bei der Berechnung ohne instationäre Neubildung kein Zusammenhang zwischen Messung und Berechnungsergebnis erkennbar.

\section{Diskussion und Schlussfolgerungen}

Das im Rahmen der Grundwasserstudie Düsseldorf entwickelte und in das Programmsystem SPRING implementierte Modell zur Ermittlung flächendifferenzierter, tagesscharfer Wasserbilanzen (RUBINFLUX) setzt den Fokus auf den grundwasserneubildungswirksamen Anteil des Niederschlags. Dieser Anteil entspricht der Wassermenge, die im Grundwassermodell als Randbedingung 2. Art für die Grundwasseroberfläche jedes Netz-Elements angesetzt wird. Laterale Wasserflüsse werden ausschließlich in der gesättigten Zone und zwischen Vorflutern und dem Grundwasser gerechnet. Die Wasserbilanz der Geländeoberfläche und der ungesättigten Zone wurde durch das modifizierte SCS-Verfahren und die Methode RUBINFLUX implizit quantifiziert, jedoch nicht implizit 2-dimensional abgebildet. In der spezifischen modellinternen Kopplung lateraler und vertikaler Wasserflüsse liegt eine Besonderheit des Modells. Die laterale Kopplung erfolgt hier indirekt durch die Interaktion zwischen dem Wasserstand im Vorfluter und dem angrenzenden Grundwasser - und damit angepasst an die Aufgabenstellung einer Grundwassermodellierung. Die gute Übereinstimmung zwischen den berechneten Tagesgängen der Wasserbilanz und den am Pegel gemessenen Abflüssen überrascht, wenn man sich vergegenwärtigt, dass unser Modell im Unterschied zu N-A-Modellen keine Algorithmen zur Dämpfung und Verzögerung unterschiedlich schneller Abflusskomponenten oberhalb der Grundwasseroberfläche besitzt. Offenbar bildet unser Ansatz einer in das Grundwassermodell integrierten Bilanzierung von Grundwasserneubildung und Direktabfluss die hydrologische Dynamik treffend ab. Im niederschlagsreichen Bergland fördert die relativ hohe Dichte an Niederschlagsstationen zu einem großen Teil die Modellgüte.
Andere Verfahren begegnen der Herausforderung, schnellen Abfluss zu berücksichtigen, indem sie die Gesamtabflusshöhe mit dem Baseflow-Index (BFI) multiplizieren (Herrmann et al. 2013; Gudera und Morhard 2015). Dieser muss in vorgeschalteten, empirischen Analysen aus langjährigen Messreihen des Vorfluterabflusses ermittelt werden. In unserem Fall ist das nicht notwendig.

Die Gras-Referenz-Verdunstung, und somit eine auf MONTEITH zurückgehende physikalische Behandlung der Evapotranspiration ist Bestandteil zahlreicher Grundwasserneubildungsermittlungen (z. B. Bundesanstalt für Gewässerkunde 2003; Meßer et al. 2013; Herrmann et al. 2013; Gudera und Morhard 2015 oder auch im Programmmodul „SIWA onArcView“ der DHI-Wasy GmbH, Berlin, einer Programmkomponente von ArcEGMO). Diese Methode entspricht dem Stand der Technik und wurde deshalb in die RUBINFLUX-Methodik aufgenommen.

Sickerwasserabgabe an den tieferen Untergrund als Funktion der aktuellen Bodenfeuchte lassen die meisten der für räumlich differenzierende Anwendungen konzipierten Verfahren erst oberhalb der Feldkapazitätsgrenze zu. Wird sie als starre Grenze behandelt, treten Sickerwasserschübe ruckhaft auf; derartige Verfahren sind empfindlich gegenüber Fehleinschätzungen der Feldkapazität. Lediglich mGROWA (Herrmann et al. 2013) und GWN-BW (Gudera und Morhard 2015) berücksichtigen, dass Grundwasserneubildung unterhalb der Feldkapazität auftreten kann, jedoch unterscheiden sich RUBINFLUX und die beiden genannten Verfahren hinsichtlich der Formulierung für diesen Prozess. Ein Vorzug von RUBINFLUX liegt in der Unempfindlichkeit gegenüber Fehleinschätzungen der Feldkapazität; unabhängig vom absoluten Wert steuert allein die Differenz des tagesaktuell simulierten Wassergehaltes zur Feldkapazität die Wasserabgabe aus dem Boden. Beiden Verfahren - GWN-BW und RUBINFLUX - ist gemeinsam, dass sie an Lysimetermessreihen plausibilisiert sind.

Die RUBINFLUX-Funktion (Gl. 4-6) sieht keine Differenzierung nach Bodenarten vor, wiewohl sich doch nach theoretischen Überlegungen auf der Grundlage der Richards-Gleichung - die Sickerwasserabgabe aus verschiedenen Böden deutlich voneinander unterscheiden sollte. Allerdings lassen sich mit dem gewählten Ansatz tatsächlich die Durchsickerungen sowohl des Sand- als auch des lössbürtigen Schluffbodens so zufriedenstellend abbilden, wie es für die Anforderungen an die regionale Grundwasserströmungsmodellierung erforderlich ist. Dies liegt zum einen an der oben erwähnten Fehlertoleranz und zum anderen an der mit bodenphysikalischen Erwägungen übereinstimmenden, nichtlinearen Abnahme der Sickerwasserabgabe bei Wassergehaltsabnahme von Böden. Dies leistet einzig die RUBINFLUX-Methodik. Es ist nicht auszuschließen, dass an den Lysimeterstandorten über die Veränderungen von 
Bestandskoeffizienten für die Verdunstungsberechnung zugleich bodenabhängige Effekte mit angepasst wurden. Die Bestandskoeffizienten im nutzungsdifferenzierten $708 \mathrm{~km}^{2}$ großen Untersuchungsraum können gleichwohl nur abgeschätzt werden, sodass hier mit sich überlagernden Einflüssen von Boden- und Nutzungsunterschieden zu rechnen ist. Dennoch ist insgesamt die Robustheit unserer Berechnungsmethodik bemerkenswert. Für die Zukunft sind Modifikationen der Entleerungsfunktion für unterschiedliche Böden geplant, eine Entwicklung, die allerdings sehr hohe Ansprüche an zeitlich hochaufgelöste Lysimetermessreihen mit langjähriger homogener Nutzung und sehr guten Klimadaten voraussetzt. Die Unsicherheit der Schätzung von Bestandskoeffizienten für große Gebiete bliebe. Eine gesteigerte Verdunstung grundwassernaher Standorte ist zurzeit nicht im Modell implementiert, diese Rückkopplung müsste ebenfalls instationär realisiert werden.

In der Literatur konnten nur wenige Beispiele (z. B. Rödiger et al. 2009) für die Qualität simulierter Tagesgänge der Grundwasseroberfläche in Hydrosystemen gefunden werden, in denen die instationäre Neubildung derart bedeutsam ist wie in dem hier vorgestellten Untersuchungsgebiet. Ohne dass RUBINFLUX-Parameter adjustiert werden mussten, hat sich diese in das Programmsystem SPRING implementierte Methodik als geeignet erwiesen, komplexe Hydro-Systeme abzubilden. Der Wert der Methode zeigte sich während der Kalibrierung des Grundwassermodells. Andernfalls hätten sich Fehler in den Neubildungsraten durch unplausible Kalibrierergebnisse - insbesondere in den Durchlässigkeitsbeiwerten und ggf. in den grundwasserbürtigen Gewässerabflüssen - ergeben. Derartige Fehler werden während der Kalibrierung eines Grundwasserströmungsmodells umso größer, je höher die Anforderungen an die zeitliche Auflösung der Strömungsprozesse sind. Vereinfacht gilt: Unplausible Grundwasserneubildungsraten führen zu keiner sinnvollen Abbildung der Grundwasserströmung bei gleichzeitiger Einhaltung plausibler hydrogeologischer Parameter. Unsere Ergebnisse belegen eindeutig, dass die Annahme konstanter jährlicher Grundwasserneubildungsraten im Bergland den Anforderungen an eine realistische Abbildung des hydrologischen Systems ebenso wenig gerecht wird wie pauschale, räumlich undifferenzierte Zuflüsse über einen Modellrand. Die Modellinnovation besteht in der Integration instationärer hydrologischer Prozesse an der Geländeoberfläche, in der ungesättigten Zone, im Vorflutersystem und im Grundwasserkörper. Nunmehr beschreibt das GW-Strömungsmodell SPRING die Interaktion zwischen Vorfluter und Grundwasser im Bereich von Fest- und Lockergesteinsaquiferen, integriert darüber hinaus den an die Vorfluter gekoppelten, instationären Direktabfluss und die implizite räumlich differenzierte, instationäre Berechnung der Grundwasserneubildung nach der neuartigen Methodik RUBINFLUX.
Danksagung Die Ergebnisse sind Teil des Projekts der Landeshauptstadt Düsseldorf „Grundwasserstandsentwicklung infolge des Klimawandels am Beispiel der Stadt Düsseldorf". Wir danken dem Rheinisch-Bergischen Wasserverband (BRW) für die Überlassung von Landnutzungs-, Klima- und Abflussdaten sowie Daten zum Gewässersystem. Der Ruhrverband überließ uns freundlicherweise Pegeldaten der Ruhr. Die NEW Niederrhein Energie und Wasser (Detlef Schumacher) stellte dankenswerterweise Lysimeter- und Klima-Messreihen der Hydrologischen Station Rheindahlen zur Verfügung, und dem Landesamt für Natur, Umwelt und Verbraucherschutz NordrheinWestfalen danken wir für die Erlaubnis, Modelltests an den Lysimeterund Klima-Messreihen St. Arnold durchzuführen. Weitere, allgemein zugängliche Daten stammen von öffentlichen Dienststellen (Deutscher Wetterdienst, Geologischer Dienst NRW, Bezirksregierung Köln) sowie der Stadt Düsseldorf.

Open Access Dieser Artikel wird unter der Creative Commons Namensnennung 4.0 International Lizenz (http://creativecommons.org/ licenses/by/4.0/deed.de) veröffentlicht, welche die Nutzung, Vervielfältigung, Bearbeitung, Verbreitung und Wiedergabe in jeglichem Medium und Format erlaubt, sofern Sie den/die ursprünglichen Autor(en) und die Quelle ordnungsgemäß nennen, einen Link zur Creative Commons Lizenz beifügen und angeben, ob Änderungen vorgenommen wurden.

\section{Literatur}

ATV-DVWK (Deutsche Vereinigung für Wasserwirtschaft, Abwasser und Abfall) (Hrsg.): Verdunstung in Bezug zu Landnutzung, Bewuchs und Boden. Merkblatt M504, 144 S.; Hennef (2002)

Baudena, M., Bevilacqua, I., Canone, D., Ferraris, S., Previati, M., Provenzale, A.: Soil water dynamics at a midlatitude test site: field measurements and box modeling approaches. J Hydrol 414-415, 329-340 (2012)

Becker, M., Rosemann, H.J.: Regionalisierung von Hochwasserabflüssen für kleine Einzugsgebiete in Bayern. Ein Beitrag zur Bereitstellung hydrologischer Planungsgrundlagen. Hydraulik und Gewässerkunde 61. TU München, Institut für Wasserwesen, München (1995)

Berthold, G., Hergesell, M.: Flächendifferenzierte Untersuchungen zu möglichen Auswirkungen einer Klimaänderung auf die Grundwasserneubildung in Hessen. INKLIM 2012 (Integriertes Klimaschutzprogramm Klimafolgen in der Wasserwirtschaft (Grundwasser). Abschlussbericht für den Bereich Grundwasser. Hessisches Landesamt für Umwelt und Geologie, Wiesbaden, $21 \mathrm{~S}$. (2005)

Bogena, R., Kunkel, T., Schöbel, H., Schrey, R., Wendland, E.: Die Grundwasserneubildung in Nordrhein-Westfalen. Schriften des Forschungszentrums Jülich Reihe Umwelt/Environment, Bd. 37. Forschungszentrum Jülich, Jülich (2003)

Bundesanstalt für Gewässerkunde (Hg.): Wasserhaushaltsverfahren zur Berechnung vieljähriger Mittelwerte der tatsächlichen Verdunstung und des Gesamtabflusses. BfG-Bericht Nr. 1342, Koblenz (2003)

Disse, M.: Modellierung der Verdunstung und der Grundwasserneubildung in ebenen Einzugsgebieten. Mitteilungen des Instituts für Hydrologie und Wasserwirtschaft der Universität Karlsruhe (TH) 53. Institut für Hydrologie und Wasserwirtschaft der Universität Karlsruhe, Karlsruhe, S. 243 (1995)

Dörhöfer, G., Josopait, V.: Eine Methode zur flächendifferenzierten Ermittlung der Grundwasserneubildung. Geol Jahrb C27, 45-65 (1980)

DVWK: Beitrag zur Bestimmung des effektiven Niederschlags für Bemessungshochwasser aus den Gebietskenngrößen. DVWK-Materialien 2. Deutsche Vereinigung für Wasserwirtschaft, Abwasser und Abfall, Hennef, Bonn (1991) 
Hoyningen-Huene, J.: Die Interzeption des Niederschlags in landwirtschaftlichen Pflanzenbeständen. DVWK Schriften 57., S. 1-53 (1983)

FAO (Food and Agriculture Organization of the United Nations): ETo calculator (version 3.2). Rome (2012). http://www.fao.org/nr/ water/eto.html, Zugegriffen: 05. Jan 2016

Glugla, G., Enderlein, R., Eyrich, A.: Das Programm RASTER - ein effektives Verfahren zur Berechnung der Grundwasserneubildung im Lockergestein. Wasserwirtsch Wassertech 26(11), 377-382 (1976)

Glugla, G., Fürtig, G.: Abflußbildung in urbanen Gebieten. Schriftenreihe Hydrologie, Wasserwirtschaft 14., S. 140-160 (1997)

Gudera, T., Morhard, A.: Hoch aufgelöste Modellierung des Bodenwasserhaushalts und der Grundwasserneubildung mit GWN-BW. Hydrol Wasserbewirtsch 59(5), 205-216 (2015). doi:10.5675/ HyWa_2015,5_1

Harsch, N., Brandenburg, M., Klemm, O.: Large-scale lysimeter site St. Arnold, Germany: analysis of 40 years of precipitation, leachate and evapotranspiration. Hydrol Earth Syst Sci 13, 305-317 (2009)

Hergesell, M., Berthold, G.: Auswirkungen des Klimawandels auf das Grundwasser in Hessen. Jahresbericht 2007 d. Hess. Landesamtes für Umwelt und Geologie, 45-53; Wiesbaden (2008). http:// www.hlug.de/fileadmin/dokumente/das_hlug/jahresbericht/2007/ 2007_045-054.pdf, Zugegriffen: 07. März 2016

Herrmann, F., Chen, S., Heidt, L., Elbracht, J., Engel, N., Kunkel, R., Müller, U., Röhm, H., Vereecken, H., Wendland, F.: Zeitlich und räumlich hochaufgelöste flächendifferenzierte Simulation des Landschaftswasserhaushalts in Niedersachsen mit dem Modell mGROWA. Hydrol Wasserbewirtsch 57, 206-224 (2013). doi:10. 5675/HyWa_2013,5_2

Klein, M.: Langjähriger Wasserhaushalt von Gras- und Waldbeständen. Entwicklung, Kalibrierung und Anwendung des Modells LYFE am Groß-Lysimeter St. Arnold. Diss. Univ. Osnabrück (2000)

Kim, C., Stricker, J., Torf, P.: An analytical framework for the water budget of the unsaturated zone. Water Resour Res 32, 3475-3484 (1996)

König, C., Becker, M., Diehl, A., Rosen, B., Rüber, O., Seidel, T., Werth, B., Zimmermann, C.: SPRING, Benutzerhandbuch, Ausgabe 4.1. delta h Ingenieurgesellschaft mbH, Witten (2012). ISBN 978-3000403699

Laio, F., Porporato, A., Ridolfi, L., Rodriguez-Iturbe, I.: Plants in water controlled ecosystems: active role in hydrological processes and response to water stress: II. Probabilistic soil moisture dynamics. Adv Water Resour 24, 707-723 (2001)

Lang, U., Justiz, J., Morhard, A., Gudera, T., Emmert, M.: Auswirkungen des Klimawandels auf die Entwicklung der Nitratbelastung im Grundwasser am Beispiel des Gesamteinzugsgebiets des Donaurieds. Forschungsbericht KLIMOPASS. LUBW Landesanstalt für Umwelt, Messungen und Naturschutz Baden-Württemberg (Hg.), $150 \mathrm{~S}$ (2012). http://www.fachdokumente.lubw. baden-wuerttemberg.de/servlet/is/107182/U62-W03-N10.pdf? command=downloadContent\&filename=U62-W03-N10.pdf, Zugegriffen: 07. März 2016

Manfreda, S., Fiorentino, M., Lacobellis, V.: DREAM: a distributed model for runoff, evapotranspiration, and antecedent soil moisture simulation. Adv Geosci 2, 31-39 (2005)

Menzel, L.: Flächenhafte Modellierung der Evapotranspiration mit TRAIN. Potsdam Institute of Climate Impact Research (PIK) Report 54, 30 S (1999). https://www.pik-potsdam.de/research/ publications/pikreports/.files/pr54.pdf, Zugegriffen: 07. März 2016

Meßer, J.: Auswirkungen der Urbanisierung auf die Grundwasserneubildung im Ruhrgebiet unter besonderer Berücksichtigung der Ca- stroper Hochfläche und des Stadtgebietes Herne. Diss. TU Clausthal-Zellerfeld. (1996)

Meßer, J., Ohlenbusch, R., Getta, M.: Klimawandel-bedingte Veränderungen der Grundwasserneubildung im urbanen Raum am Beispiel des Emschergebietes. dynaklim-Publikation 33, $24 \mathrm{~S}$ (2013). http://opus.kobv.de/zlb/volltexte/2013/20923/pdf/33_ dynaklimKlimawandel.pdf, Zugegriffen: 07. März 2016

Milly, P.C.D.: A minimalist probabilistic description of root zone soil water. Water Resour Res 37, 457-464 (2001)

Regionaler Planungsverband Leipzig-Westsachsen: Auswirkungen des Klimawandels auf den Südraum Leipzig unter besonderer Berücksichtigung der Auswirkungen auf den Wasserhaushalt und die Erholungsnutzungen sowie der Anforderungen an Waldumbau und Waldmehrung. Modellvorhaben der Raumordnung (MORO) „Raumentwicklungsstrategien zum Klimawandel“. Endbericht, 30 S (2013). http://www.klimamoro.de/fileadmin/Dateien/Ver \%C3\%B6ffentlichungen/Ver\%C3\%B6ffentlichungen_Phase_II/ Endbericht_30_04_2013.pdf, Zugegriffen: 07. März 2016

Renger, M., Strebel, O.: Jährliche Grundwasserneubildung in Abhängigkeit von Bodennutzung und Bodeneigenschaften. Wasser Boden 32(8), 362-366 (1980)

Renger, M., Wessolek, G.: Berechnung der Verdunstungs-Jahressummen einzelner Jahre. DVWK-Merkblätter zur Wasserwirtschaft 238. Gesellschaft zur Förderung der Abwassertechnik e.V. (GFA), Bonn, 47 S. (1996)

Rödiger, T., Sauter, M., Büchel, G.: Infiltration und Grundwasserströmung in geklüftet-porösen Buntsandsteingrundwasserleitern im Osten des Thüringer Beckens. Grundwasser 14(1), 21-32 (2009)

Schröder, M., Wyrwich, D.: Eine in Nordrhein-Westfalen angewandte Methode zur flächendifferenzierten Ermittlung der Grundwasserneubildung. Dtsch Geol Mitteilungen 34(1/2), 12-16 (1990)

Schumacher, D., Wellens, M.: Zehn Jahre Hydrologische Station Mönchengladbach-Rheindahlen (1983-1992). Stadtwerke Mönchengladbach, Mönchengladbach (1993)

Sheffer, N.A., Dafny, E., Gvirtzman, H., Navon, S., Frumkin, A., Morin, E.: Hydrometeorological daily recharge assessment model (DREAM) for the Western Mountain Aquifer, Israel: Model application and effects of temporal patterns. Water Resour Res 46, W05510 (2010). doi:10.1029/2008WR007607

Šimůnek, J., Šejna, M., van Genuchten, M.Th.: The HYDRUS-1D software package for simulating the one-dimensional movement of water, heat, and multiple solutes in variably- saturated media. Version 1.0. IGWMC - TPS - 70. International Ground Water Modeling Center, Colorado School of Mines, Golden (1998)

Šimůnek, J., van Genuchten, M.T., Šejna, M.: The HYDRUS software package for simulating two- and three-dimensional movement of water, heat, and multiple solutes in variably-saturated media; technical manual, version 1.0. PC Progress, Prague (2006)

Stadt Düsseldorf: Risikokataster für Nässeschäden (2009). https:// www.duesseldorf.de/umweltamt/service/presse19/archiv_2009/ p19_2009_august_24.shtm, Zugegriffen: 30. Dez 2015

Ullrich, K.: Grundwasserbewirtschaftung und Klimawandel. Handlungsansätze für Planungsträger und Genehmigungsbehörden. REGKLAM-Teilprojekt 3.2.2: Wasserhaushalt Stadt - Umland, Produkt 3.2.2 e. REGKLAM Ergebnisbericht (Landeshauptstadt Dresden (Hg.), 28.S (2013). http://www.regklam.de/ fileadmin/Daten_Redaktion/Publikationen/Ergebnisberichte/P3. 2.2e_Bewirtschaftungsleitfaden_v1.0.pdf, Zugegriffen: 30. Dez 2015

Williams, J.R.: The EPIC model. In: Singh, V.P. (Hrsg.) Computer models of watershed hydrology, S. 909-1000. Water Resources Publications, Highlands Ranch (1995) 